\title{
Specificity protein 1-activated bone marrow stromal cell antigen 2 accelerates pancreatic cancer cell proliferation and migration
}

\author{
CHUN LEI ${ }^{1-3}$, YAFENG HOU ${ }^{1-3}$ and JIONG CHEN ${ }^{4}$ \\ ${ }^{1}$ Department of General Surgery, Tongling People's Hospital; ${ }^{2}$ Department of General Surgery, Tongling People's Hospital \\ Affiliated to Wannan Medical College; ${ }^{3}$ Department of General Surgery, Tongling Branch of the First Affiliated Hospital of \\ University of Science and Technology of China, Tongling, Anhui 244009; ${ }^{4}$ Department of General Surgery, \\ The First Affiliated Hospital of University of Science and Technology of China \\ (Anhui Provincial Hospital), Hefei, Anhui 230001, P.R. China
}

Received July 15, 2021; Accepted September 14, 2021

DOI: 10.3892/etm.2021.10894

\begin{abstract}
Bone marrow stromal cell antigen 2 (BST2) has been reported to act as an oncogene in the tumorigenesis of numerous types of cancer. Bioinformatics analysis has predicted the binding interaction between BST2 and specificity protein 1 (SP1) and the involvement of SP1 in pancreatic cancer. Therefore, the present study set out to verify this interaction and determine how it may affect pancreatic cancer progression. Normal human pancreatic duct epithelial cells (HPDE6-C7) and pancreatic cancer cell lines (SW1990, BxPC3, PANC1 and PSN-1) were selected for western blotting and reverse transcription-quantitative PCR detection of BST2 expression. Colony formation, Cell Counting Kit-8 and wound healing assays were performed to detect the proliferative and migratory abilities of PANC1 cells following transfection with small interfering RNA against BST2. The expression of proliferation and migration markers were assayed using western blotting. Chromatin immunoprecipitation and luciferase reporter assays were employed to verify the bioinformatics prediction of BST2-SP1 binding. PANC1 cell proliferation and migration were analyzed following BST2 knockdown and SP1 overexpression. In comparison with HPDE6-C7 cells, all four pancreatic cancer cell lines were found to exhibit increased BST2 expression levels to varying degrees, with the highest levels observed in PANC1 cells. BST2 knockdown inhibited PANC1 cell colony formation, proliferation and migration. Additionally, SP1 was shown to bind to the BST2 promoter and could promote PANC1 cell proliferation and migration when overexpressed. However, BST2 knockdown rescued SP1 overexpression-induced
\end{abstract}

Correspondence to: Dr Chun Lei, Department of General Surgery, Tongling People's Hospital, 468 Bijiashan Road, Tongling, Anhui 244009, P.R. China

E-mail: leichun518@126.com

Key words: pancreatic cancer, specificity protein 1, bone marrow stromal cell antigen 2, proliferation, migration
PANC1 cell colony formation, proliferation and migration. In conclusion, activation of BST2 by the transcription factor SP1 was shown to accelerate pancreatic cancer cell proliferation and migration, suggesting that BST2 and SP1 may be plausible therapeutic targets in targeted therapy for pancreatic cancer.

\section{Introduction}

Pancreatic cancer is a common malignancy of the digestive system and usually presents as ductal adenocarcinoma originating from the pancreatic duct epithelium (1). The incidence of pancreatic cancer has been on the rise due to the lifestyle changes in modern society (2). The current understanding of pancreatic carcinogenesis is limited, but habits such as cigarette smoking, alcohol intake, high-fat diet and excessive consumption of caffeine have been identified as some of the contributing factors (3). The 5-year survival rate of pancreatic cancer after diagnosis is reportedly as low as $\sim 9 \%$, rendering it one of the malignancies with the poorest prognosis $(4,5)$. In addition to the strong propensity of pancreatic cancer for metastasis, patients with early-stage disease are often asymptomatic; both these factors contribute to the high mortality rate of this malignancy and, in numerous cases, delay diagnosis until the disease is at an advanced stage, when effective treatment options are limited (6-8). In recent years, gene therapy based on gene signal transduction suppression for pancreatic cancer has entered the clinical trial stage as an innovative treatment (9). The screening of core genes in pancreatic cancer in combination with the development of conventional chemotherapy and radiotherapy is essential for accurate targeting of the oncogenes in gene therapy $(10,11)$.

Bone marrow stromal cell antigen 2 (BST2), also known as CD317/tetherin, is a type II transmembrane protein that is widely expressed by bone marrow stromal cells, B cells, T cells and natural killer cells (12). Notably, it has been established that certain immunocytes and malignant cells, such as B-cell chronic lymphoid leukemia cells and pulmonary cancer cells, also exhibit differential expression of BST2 $(13,14)$. More importantly, the bioinformatics database Gene Expression Profiling Interactive Analysis (GEPIA; http://gepia2.cancer-pku.cn) has demonstrated BST2 upregulation in pancreatic cancer and a 
distinct association between high BST2 expression and lower overall survival. In addition, the transcription factor specificity protein 1 (SP1) has been shown to play a key role in pancreatic cancer, and high SP1 expression has been reported to be a key tumorigenic factor by several studies on pancreatic tumorigenesis (15-17). Thus, the present study was undertaken to investigate the association of BST2 with pancreatic cancer occurrence and development, and determine whether transcriptional regulation by SP1 is involved in this process.

\section{Materials and methods}

Cell culture and treatment. A human pancreatic duct epithelial cell line (HPDE6-C7) and pancreatic cancer cell lines (SW1990, BxPC3, PANC1 and PSN-1) were purchased from EK-Biosciences GmbH. HPDE6-C7 cells were cultured in $89 \%$ DMEM supplemented with $10 \%$ FBS and $1 \%$ penicillin-streptomycin solution(P/S); SW1990 cells were cultured in Leibovitz L15 medium supplemented with $10 \% \mathrm{FBS}$ and $1 \% \mathrm{P} / \mathrm{S}$ (in an environment free of $\mathrm{CO}_{2}$ at $37^{\circ} \mathrm{C}$ ); $\mathrm{BxPC} 3$ cells were cultured in RPMI-1640 medium supplemented with $10 \%$ FBS and $1 \% \mathrm{P} / \mathrm{S}$; and PANC1 and PSN-1 cells were cultured in RPMI-1640 medium supplemented with $15 \%$ FBS, $0.01 \mathrm{mg} / \mathrm{ml}$ insulin and $1 \% \mathrm{P} / \mathrm{S}$. All the aforementioned reagents were purchased from Thermo Fisher Scientific, Inc.

Cell transfection. Small interfering RNA (siRNA) plasmids specific for BST2 (siBST2-1: 5'-GGCATCTACTTG TATGACTATT-3'; siBST2-2: 5'-TCCTTTGGATGGCCT AGTACTAG-3'), empty siRNA vector negative control (siNC: 5'-GTAAGCTTCTGCTGGGGATAGG-3'), wild-type BST2 (BST2-WT) (5'-CAGGCCCCGCCCCCA-3') and mutant BST2 (BST2-MUT) (5'-CAAGCCGGAGGUUUG-3') promoter, siRNA plasmids targeting SP1 (siSP1-1: 5'-CCGAAACC TTCTGACTACTAACC-3'; siSP1-2: 5'-ATGCCTAATAT TC AGTATCAAGT-3'), pcDNA 3.1(+)/SP1 [overexpression (OE)-SP1)] and empty pcDNA 3.1(+) vectors were constructed by Guangzhou RiboBio Co., Ltd. PANC-1 cell transfection was performed using Lipofectamine ${ }^{\circledR} 2000$ (Invitrogen; Thermo Fisher Scientific, Inc.) following the manufacturer's protocol. Cells were harvested following $48 \mathrm{~h}$ of transfection at $37^{\circ} \mathrm{C}$ and the transfection efficiency was determined using RT-qPCR. The subsequent experimentation was conducted within $48 \mathrm{~h}$.

Reverse transcription-quantitative PCR (RT-qPCR) analysis. Total RNA was extracted from transfected cells by means of the spin column-based method using the MolPure ${ }^{\circledR}$ Cell RNA kit (Shanghai Yeasen Biotechnology Co., Ltd.). Subsequently, cDNA was synthesized at $42^{\circ} \mathrm{C}$ for 30 min using a PrimeScript ${ }^{\mathrm{TM}}$ RT Reagent kit (Takara Bio, Inc.). The PCR system was set in accordance with the instructions of BeyoFast ${ }^{\mathrm{TM}}$ Probe qPCR Mix (Beyotime Institute of Biotechnology). After pre-denaturation and amplification of the template (initial denaturation at $95^{\circ} \mathrm{C}$ for $10 \mathrm{~min}$; followed by 40 cycles of denaturation at $95^{\circ} \mathrm{C}$ for $15 \mathrm{sec}$ and annealing at $60^{\circ} \mathrm{C}$ for $1 \mathrm{~min}$; and a final extension of $10 \mathrm{~min}$ at $72^{\circ} \mathrm{C}$ ), the results were analyzed using software provided by a fluorescent quantitative PCR instrument (Applied Biosystems; Thermo Fisher Scientific, Inc.). The $2^{-\Delta \Delta \mathrm{Cq}}$ method was used to compare relative expression levels (18). The following primer pairs were used: BST2 forward, 5'-AGCGACTGAGAAGAGAAA ACCA-3' and reverse, 5'-TGTTCAAGCGAAAAGCCGAG-3'; and U6 forward, 5'-AAAGCAAATCATCGGACGACC-3' and reverse: 5'-GTACAACACATTGTTTCCTCGGA-3'.

Western blotting. Total proteins were extracted from transfected cells using the RIPA lysis buffer (Shanghai Yeasen Biotechnology Co., Ltd.) followed by protein quantification using a BCA kit (Shanghai Enzyme-linked Biotechnology Co., Ltd.) and protein separation was performed on $10 \%$ gels using SDS-PAGE. PVDF membranes containing the proteins were blocked using 5\% skimmed milk for $1 \mathrm{~h}$ at room temperature and incubated with anti-BST2 (1:1,000; cat no. ab243230), anti-SP1 (1:1,000; cat no. ab227383), antiKi67 (1:1,000; cat no. ab16667), anti-proliferating cell nuclear antigen (anti-PCNA; 1:1,000; cat no. ab18197), anti-MMP2 (1:1,000; cat no. ab92536) and anti-MMP9 (1:1,000; cat no. ab38898) primary antibodies (all from Abcam) at $4^{\circ} \mathrm{C}$ overnight. Mouse anti-rabbit IgG HRP-conjugated secondary antibody (1:1,000; cat no. sc-2357; Santa Cruz Biotechnology, Inc.) was used for subsequent incubation with $2 \mathrm{~h}$ at room temperature. Protein bands were visualized using Immobilon ${ }^{\mathrm{TM}}$ Chemiluminescent HRP substrate (MilliporeSigma).

Analysis of cell proliferation. To evaluate cell colony formation ability, transfected cells were plated into a 6 -well plate $\left(1 \times 10^{4}\right.$ cells/well) and the cells were allowed to grow for 10 days at $37^{\circ} \mathrm{C}$. The colonies were observed after $3.7 \%$ paraformaldehyde fixation for $10 \mathrm{~min}$ at room temperature and $0.2 \%$ crystal violet staining (Shanghai Aladdin Biochemical Technology Co., Ltd.) for $5 \mathrm{~min}$ at room temperature.

A Cell Counting Kit-8 (CCK-8) assay (Beijing Solarbio Science \& Technology Co., Ltd.) was used to assess cell proliferation. Briefly, transfected cells were incubated with CCK-8 solution for another $4 \mathrm{~h}$ at $37^{\circ} \mathrm{C}$ before the reading of $\mathrm{OD}_{450}$ using a microplate reader (Bio-Rad Laboratories, Inc.).

Analysis of cell migration. Transfected cells ( $3 \times 10^{5}$ cells/well) were cultured in 6-well culture plates to achieve $80-90 \%$ confluence. The cells were then incubated overnight at $37^{\circ} \mathrm{C}$ with serum-free RPMI-1640 medium. A linear scratch was created in the cell monolayer using the tip of a $200-\mu 1$ pipette. At 0 and $24 \mathrm{~h}$ of wound healing, cell migration was observed using a light microscope (magnification, x100).

Analysis of cell invasion. Transfected cells were seeded into the upper chamber of a Transwell insert pre-coated (at $37^{\circ} \mathrm{C}$ overnight) with Matrigel (Corning, Inc.) in serum-free RPMI-1640 medium. Complete medium containing 10\% FBS was added to the lower chamber. Following a 12-h incubation at $37^{\circ} \mathrm{C}$, the invading cells in lower chamber were fixed with $4 \%$ paraformaldehyde for $15 \mathrm{~min}$ at $37^{\circ} \mathrm{C}$, followed by staining with $0.1 \%$ crystal violet solution for $10 \mathrm{~min}$ at $37^{\circ} \mathrm{C}$. Cell invasion was evaluated by counting the cells under a light microscope (magnification, x100).

Bioinformatics analysis. The expression of BST2 in pancreatic cancer was analyzed using the GEPIA database (http://gepia2.cancer-pku.cn), which is a newly developed 
A

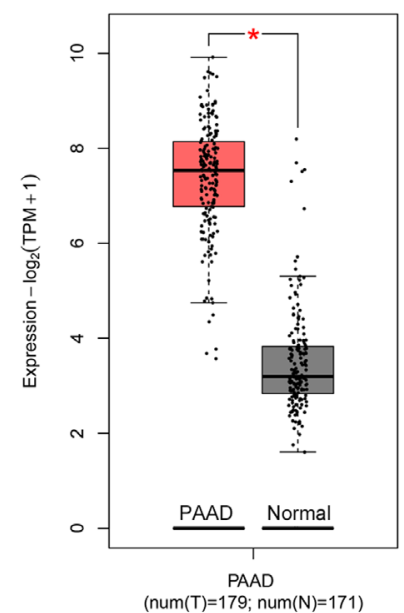

C

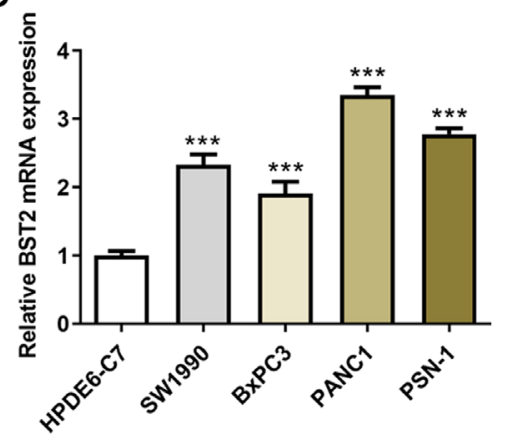

B

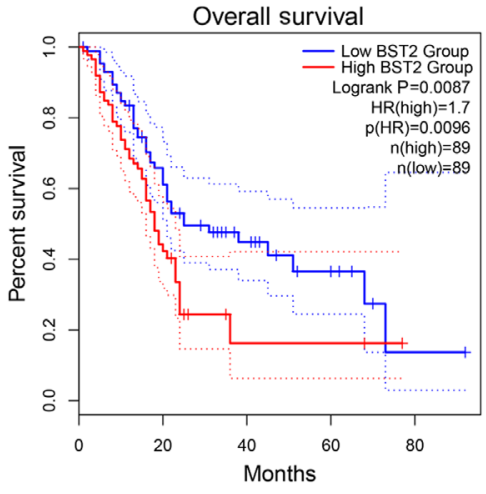

D
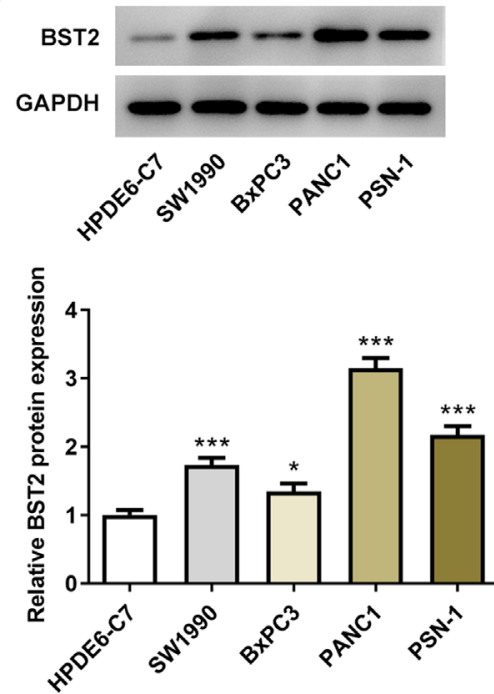

Figure 1. BST2 expression is elevated in pancreatic cancer. Gene Expression Profiling Interactive Analysis database predicted (A) the level of BST2 expression in pancreatic cancer and (B) the association between BST2 expression and overall survival. BST2 (C) mRNA and (D) protein expression levels in non-cancerous pancreatic duct epithelial cell line (HPDE6-C7) and pancreatic cancer cell lines (SW1990, BxPC3, PANC1 and PSN-1), detected by reverse transcription-quantitative PCR analysis and western blotting, respectively. ${ }^{*} \mathrm{P}<0.05,{ }^{* * *} \mathrm{P}<0.001$ vs. HPDE6-C7 cells. PAAD, pancreatic adenocarcinoma; BST2, bone marrow stromal cell antigen 2 .

interactive web server for analyzing the 9,736 tumors and 8,587 normal samples from The Cancer Genome Atlas and the Genotype-Tissue Expression project, using a standard processing pipeline. The JASPAR database 2020 (http://jaspar. genereg.net/) predicted sequence matching between the transcription factor SP1 and BST2.

Verification of SP1-BST2 binding. For chromatin immunoprecipitation (ChIP), $1 \%$ formaldehyde was added to the culture medium to fix PANC1 cells for $12 \mathrm{~min}$ at room temperature, and PBS-washed cells were harvested at $4^{\circ} \mathrm{C}$ with $300 \mathrm{x} \mathrm{g}$ for $5 \mathrm{~min}$ and resuspended in hypotonic buffer supplemented with $10 \mathrm{mM}$ ethylene diamine tetraacetic acid (EDTA). After collection of the nuclei using centrifugation at $4^{\circ} \mathrm{C}$ with $15,000 \mathrm{x} \mathrm{g}$ for $1 \mathrm{~min}$ and resuspension in the dilution buffer, $60 \mu \mathrm{l}$ Protein A/G (PrimeGene; Bio-Techne) was added to the DNA solution for antibody incubation. Following elution of the Protein A/G beads, DNA sequences binding to IgG or SP1 were detected via RT-qPCR.

For the luciferase reporter assay, PANC1 cells were co-transfected with siSP1-2 (50 nM; Guangzhou RiboBio Co., Ltd.) or si-NC (50 nM; Guangzhou RiboBio Co., Ltd.) and $50 \mathrm{ng}$ BST2-WT or BST2-MUT. Lipofectamine 2000
(Invitrogen; Thermo Fisher Scientific, Inc.) was used for transfection. After $48 \mathrm{~h}$ of transfection at $37^{\circ} \mathrm{C}$ with the luciferase reporter vector (Promega Corporation), a Dual Luciferase Reporter Assay kit (Promega Corporation) was used to evaluate the relative luciferase signals. Relative luciferase activity was expressed as the ratio of the firefly luciferase activity to that of the Renilla luciferase activity.

Statistical analysis. Experiments in this study were repeated in triplicate. The data are expressed as the mean \pm SD and were analyzed using one-way ANOVA followed by Dunnett's and Tukey's post hoc tests, as appropriate, or using an unpaired Student's t-test. $\mathrm{P}<0.05$ was considered to indicate a statistically significant difference.

\section{Results}

BST2 is highly expressed in pancreatic cancer cell lines. The GEPIA database was analyzed for 179 pancreatic adenocarcinoma samples and 171 normal pancreatic tissue samples. Based on the results of the bioinformatics analysis showing that BST2 was upregulated in pancreatic cancer (Fig. 1A) and the negative correlation between BST2 expression and overall 
A

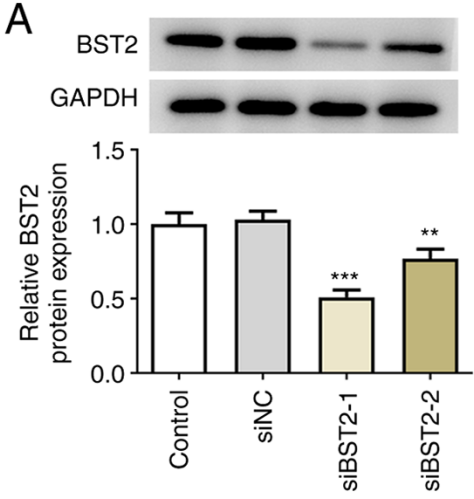

C

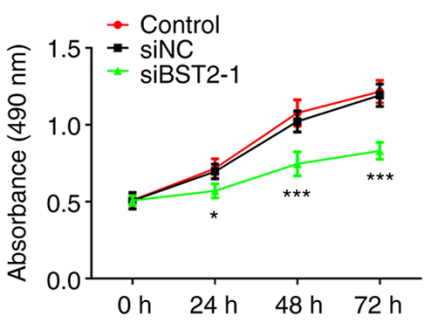

B

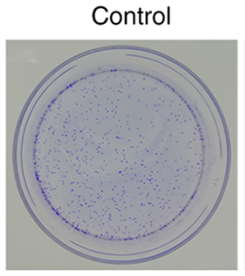

D

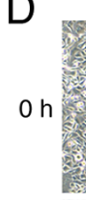

Control

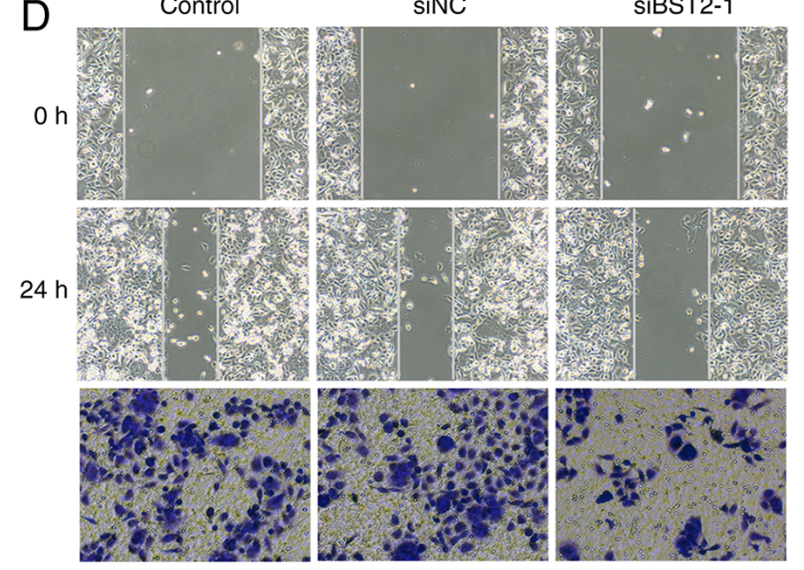

siBST2-1

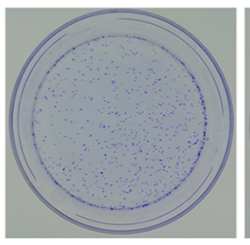

siNC

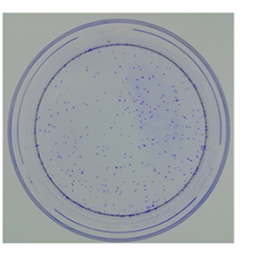

SiBST2-1
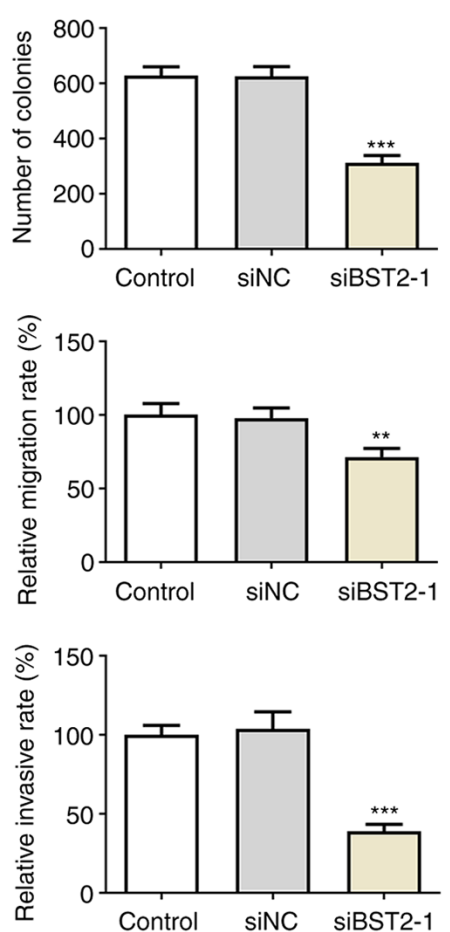

$\mathrm{E}$
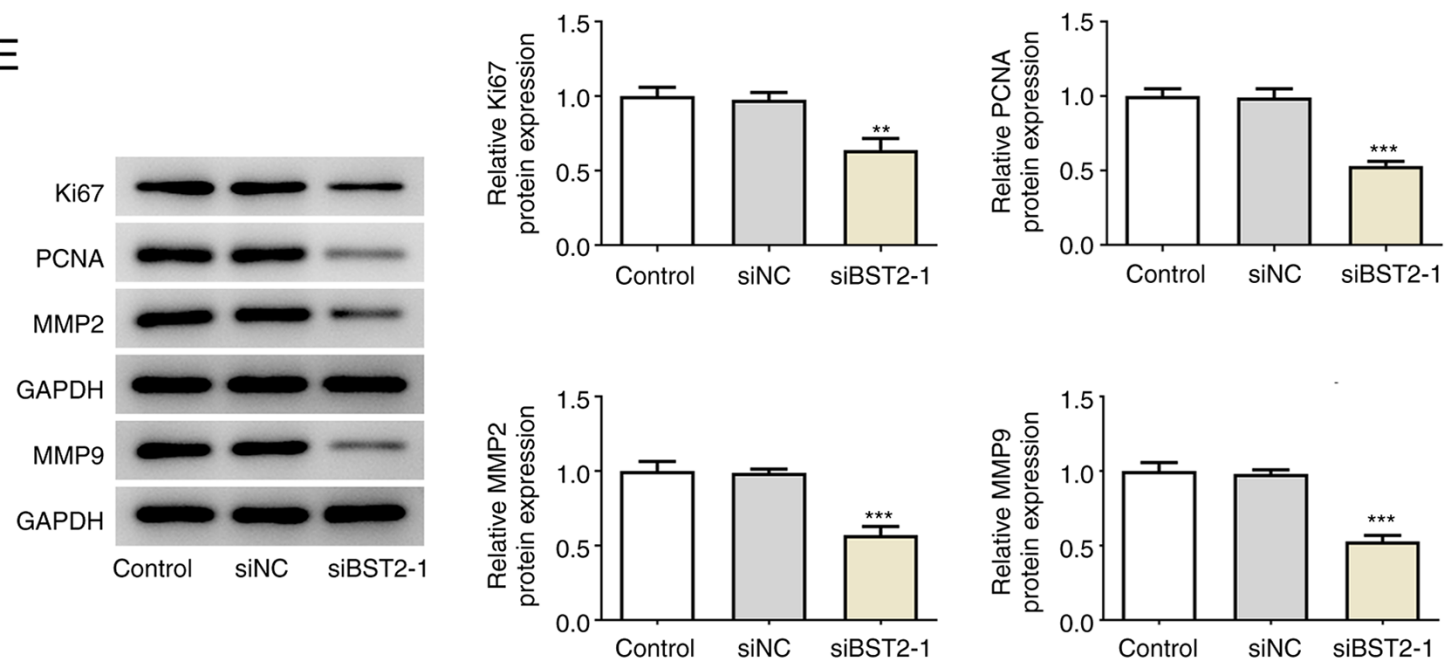

Figure 2. BST2 knockdown inhibits pancreatic cancer cell proliferation and migration. (A) Plasmid interference efficacy of siBST2-1 and siBST2-2, detected by reverse transcription-quantitative PCR analysis. (B) PANC1 colony-forming capacity before and after interference with BST2, detected by colony formation assay (magnification, x10). (C) PANC1 proliferation rate before and after interference with BST2, detected by Cell Counting Kit-8 assay. (D) Analysis of PANC1 cell migration and invasion by wound healing (top and middle rows) and Transwell (bottom row) assays, respectively (magnification, $\mathrm{x} 100$ ). (E) Proliferation and migration markers assayed by western blotting. ${ }^{*} \mathrm{P}<0.05,{ }^{* *} \mathrm{P}<0.01,{ }^{* * *} \mathrm{P}<0.001$ vs. siNC. BST2, bone marrow stromal cell antigen 2 ; si, small interfering RNA; NC, negative control.

survival (Fig. 1B), BST2 expression levels in non-cancerous pancreatic duct epithelial cells and pancreatic cancer cell lines were examined for comparison. It was observed that both the mRNA and protein expression levels of BST2 were elevated to varying degrees in SW1990, BxPC3, PANC1 and PSN-1 cells in comparison with HPDE6-C7 cells (Fig. 1C and D). Among the four pancreatic cancer cell lines, PANC1 cells exhibited the highest level of BST2 expression and were therefore selected for the subsequent experiments.

Inhibition of pancreatic cancer cell proliferation and migration by BST2 knockdown. BST2 interference was conducted by siRNA plasmid transfection in PANC1 cells, and siBST2-1 plasmid exerted a more significant knockdown effect on BST2 expression compared with siBST2-2 (Fig. 2A); hence, siBST2-1 was selected for the following assays. In PANC1 cells transfected with siBST2-1, the number of colonies was notably lower compared with that in the siNC and control groups (Fig. 2B). Moreover, CCK-8 assay detected reduced absorbance in cells transfected with siBST2-1, indicating that knockdown of BST2 reduced cell proliferation (Fig. 2C). The greater scratch width at $24 \mathrm{~h}$ in cells transfected with siBST2-1 in the wound healing assay demonstrated that BST2 knockdown could prevent pancreatic cancer cell migration to a significant extent (Fig. 2D). Furthermore, Transwell assay demonstrated that BST2 knockdown could markedly prevent pancreatic cancer 
A

\begin{tabular}{|c|c|c|c|c|c|c|c|c|c|}
\hline Matrix ID & Name & Score & Relative score & 娮 & Sequence ID & start & End & Strand & Predicted sequence \\
\hline MA0079.4 & SP1 & 19.5743 & 0.970118749044 & & NC_000019.10:C17407630-17405531 & 1588 & 1602 & + & CAGGCCCCGCCCCCA \\
\hline MA0079.4 & SP1 & 6.33596 & 0.85286069978 & & NC_000019.10:C17407630-17405531 & 1607 & 1621 & + & CAGGCCCTGCCCTCA \\
\hline
\end{tabular}

B

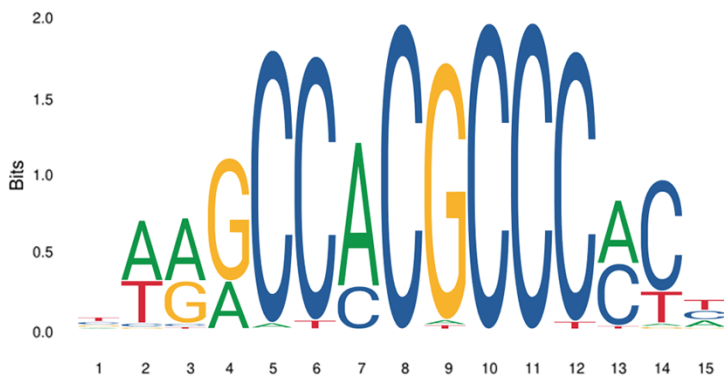

D

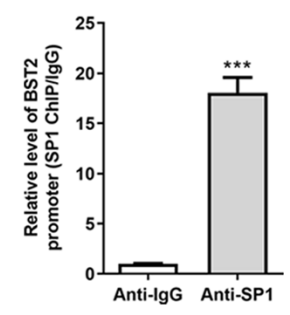

E

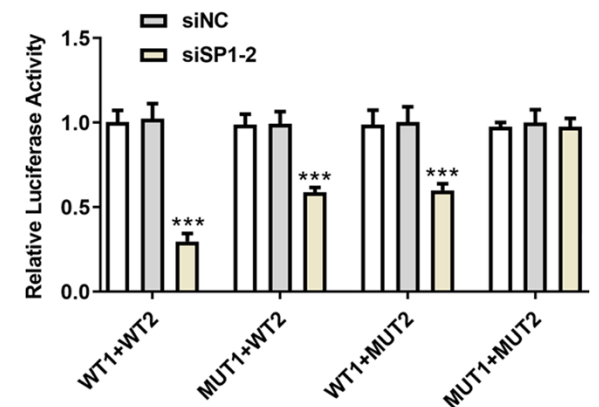

G

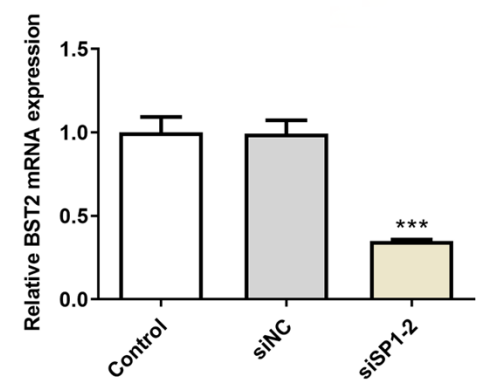

H

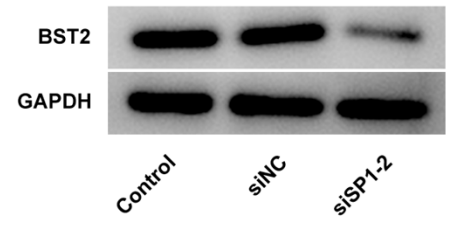

C

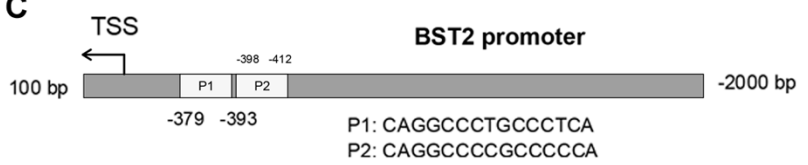

F $\quad S P 1$

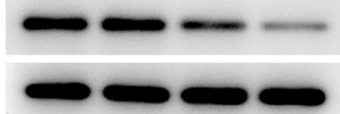

GAPDH
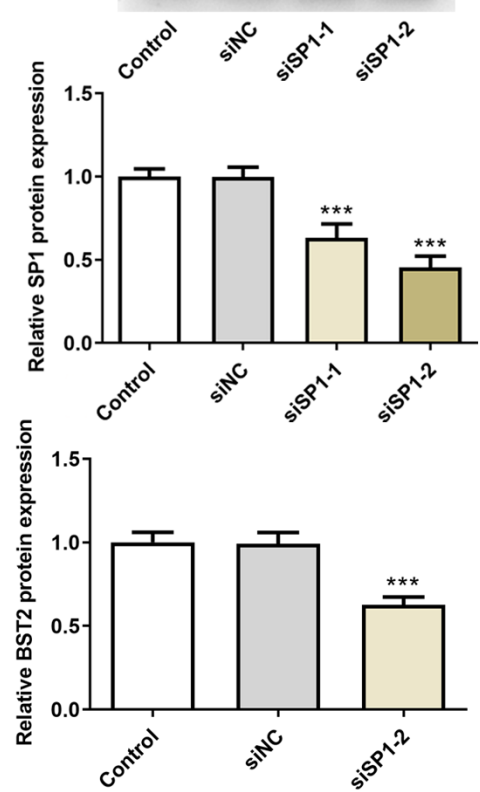

Figure 3. SP1 binds to the BST2 promoter. (A) Predicted sequence and (B) transcription factor motif of SP1 on the JASPAR database. (C) Binding sites of SP1 on the BST2 promoter. (D) Relative level of BST2 promoter detected by chromatin immunoprecipitation assay. (E) Luciferase reporter assay of the relative luciferase activity in cells co-transfected with BST2-WT/MUT and siNC/siSP1-2. (F) SP1 expression in cells transfected with siSP1-1 or siSP1-2, detected by western blotting. BST2 (G) mRNA and (H) protein expression levels in cells transfected with siNC or siSP1-2, detected by reverse transcription-quantitative PCR analysis and western blotting, respectively. ${ }^{* * *} \mathrm{P}<0.001$ vs. siNC. SP1, specificity protein 1 ; BST2, bone marrow stromal cell antigen 2 ; si, small interfering RNA; NC, negative control; WT, wild-type; MUT, mutant.

cell invasion (Fig. 2D). Additionally, the markers of proliferation (Ki67 and PCNA) and migration (MMP2 and MMP9) in PANC1 cells were found to be expressed at a markedly lower level following BST2 interference (Fig. 2E). These results collectively suggested that BST2 depletion may lead to reduced pancreatic cancer cell proliferation and migration.

Binding interaction between SP1 and BST2 promoter. The predicted sequence of SP1 (score $>5$ ) and the transcription factor DNA motif logo are shown in Fig. 3A and B. In view of the high probability of SP1 binding to the BST2 promoter (Fig. 3C),
ChIP (Fig. 3D) and dual-luciferase reporter (Fig. 3E) assays were performed for verification purposes. The results revealed a binding interaction between the SP1 and BST2 promoter. Western blotting confirmed superior interferential efficacy of siSP1-2 compared with siSP1-1 (Fig. 3F); hence, siSP1-2 was used in the dual-luciferase reporter assay. Furthermore, BST2 mRNA and protein expression levels were found to be significantly lower in the siSP1-2 group compared with those in the siNC group (Fig. 3G and H). Thus, SP1 may regulate BST2 expression in pancreatic cancer cells through their binding interaction. 
A

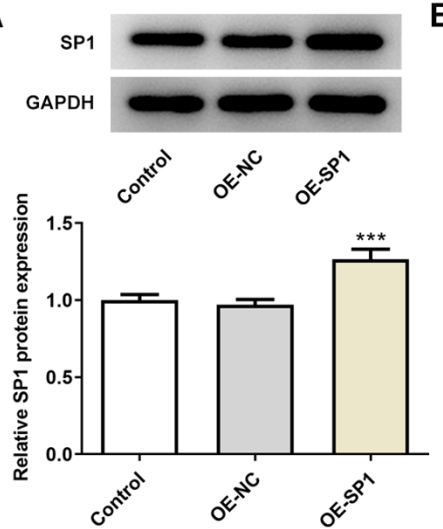

C

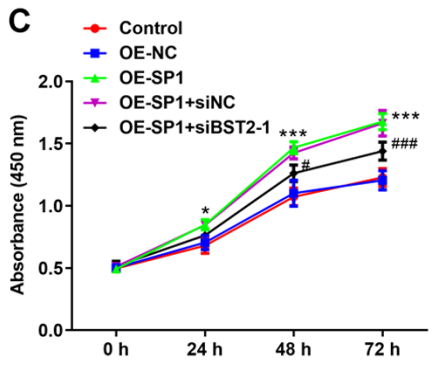

B

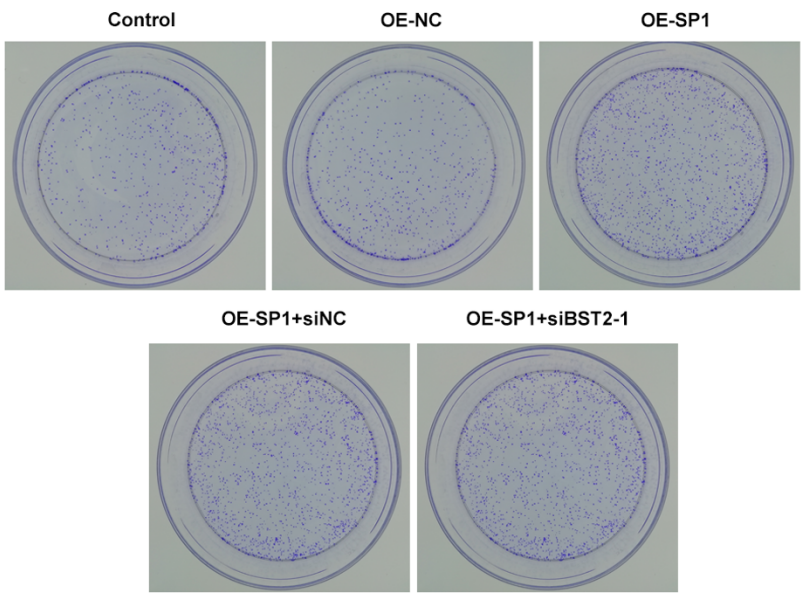

D

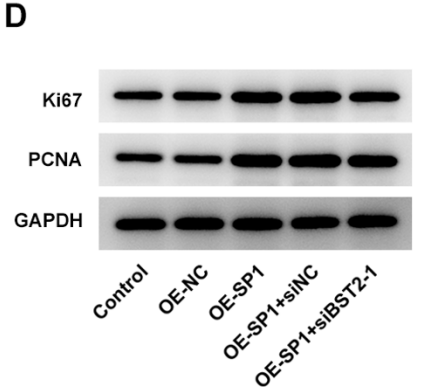

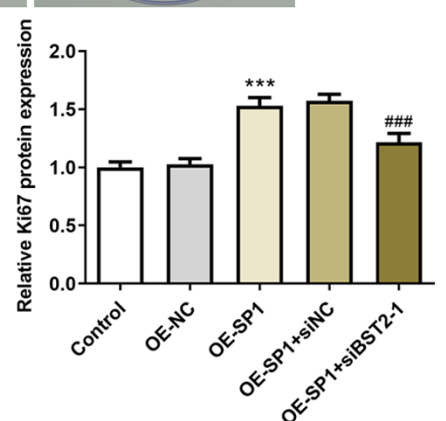
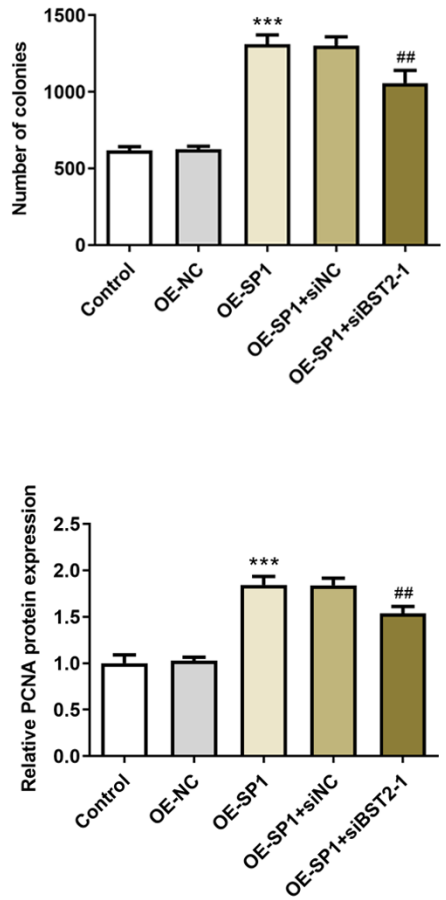

Figure 4. BST2 knockdown reverses SP1 overexpression-induced cell proliferation. (A) SP1 expression in cells transfected with OE-NC or OS-SP1, detected by western blotting. (B) PANC1 cell colony-forming capacity after transfection with OE-NC/OE-SP1 or co-transfection with OE-SP1 and siNC/siBST2-1, detected by colony formation assay (magnification, x10). (C) PANC1 cell proliferation rate after transfection with OE-NC/OE-SP1 or co-transfection with OE-SP1 and siNC/siBST2-1, detected by Cell Counting Kit- 8 assay. (D) Expression of proliferation markers assayed by western blotting. ${ }^{\text {P }<0.05}$ ${ }^{* * *} \mathrm{P}<0.001$ vs. OE-NC; ${ }^{\#} \mathrm{P}<0.05,{ }^{\# \#} \mathrm{P}<0.01,{ }^{\# \#} \mathrm{P}<0.001$ vs. OE-SP1 + siNC. SP1, specificity protein 1 ; BST2, bone marrow stromal cell antigen 2 ; OE, overexpression; NC, negative control; si, small interfering RNA; PCNA, proliferating cell nuclear antigen

SP1 overexpression-induced cell proliferation and migration are rescued by BST2 knockdown. These next assays were performed to detect the activation of BST2 by SP1 at the oncogenic transcriptional activation level in pancreatic cancer. Increased SP1 expression was verified by western blotting in PANC1 cells transfected with OE-SP1 (Fig. 4A). In subsequent colony formation assays, it was observed that the number of colonies increased after overexpression of SP1 compared with OE-NC, and that co-transfection of OE-SP1 and siBST2-1 decreased the colony number compared with the OE-SP1+siNC group (Fig. 4B). Additionally, the absorbance level $(450 \mathrm{~nm})$ was the highest in the OE-SP1 and OE-SP1+siNC groups, the lowest in the $\mathrm{OE}-\mathrm{NC}$ and control groups, and intermediate in the OE-SP1+siBST2-1 group (Fig. 4C), suggesting that the promoting effect of SP1 overexpression on PANC1 proliferation was diminished by BST2 knockdown. Furthermore, the high expression level of Ki67 and PCNA in PANC1 cells transfected with OE-SP1 declined after the knockdown of BST2 (Fig. 4D). Therefore, BST2 may be activated by SP1 in pancreatic cancer to promote cell proliferation.

Following transfection of OE-SP1, a notably higher migration rate was observed in PANC1 cells, which then decreased following interference with BST2, as shown by the results of the wound healing assay (Fig. 5A). The expression levels of migration markers, MMP2 and MMP9, which were elevated by SP1 overexpression, were also found to be downregulated by co-transfection with OE-SP1 and siBST2-1 (Fig. 5B). These results revealed a potential promoting effect of SP1-activated BST2 on pancreatic cancer cell migration.

\section{Discussion}

Pancreatic cancer is a malignant tumor originating from the pancreatic duct epithelium and it has the highest degree of malignancy and the highest rate of mortality among gastrointestinal diseases (19). Pancreatic cancer can develop local invasion and distant metastasis in its early stages; therefore, $\sim 80 \%$ of patients are at an advanced stage at the time of diagnosis $(1,20)$. Surgery is currently the only possible curative option, but radical resection is suitable for $<10 \%$ of patients $(21,22)$. It has been confirmed that the main causes of death in patients with pancreatic cancer are tumor metastasis and early recurrence (23). Therefore, the focus of pancreatic cancer research is to identify tumor markers and therapeutic molecular targets associated with pancreatic cancer metastasis and to further elucidate the biological mechanisms implicated in tumor development.

BST2 is expressed in not only normal human tissues, but also in a variety of tumor cells, and is involved in the regulation of cancer cell proliferation, migration and invasion (24). A microarray analytic data-driven study reported that tetraspanin-8 and BST2 were expressed at abnormally high levels in CD166-positive pancreatic cancer cell lines and in a mouse model with significantly accelerated tumor growth (25). 

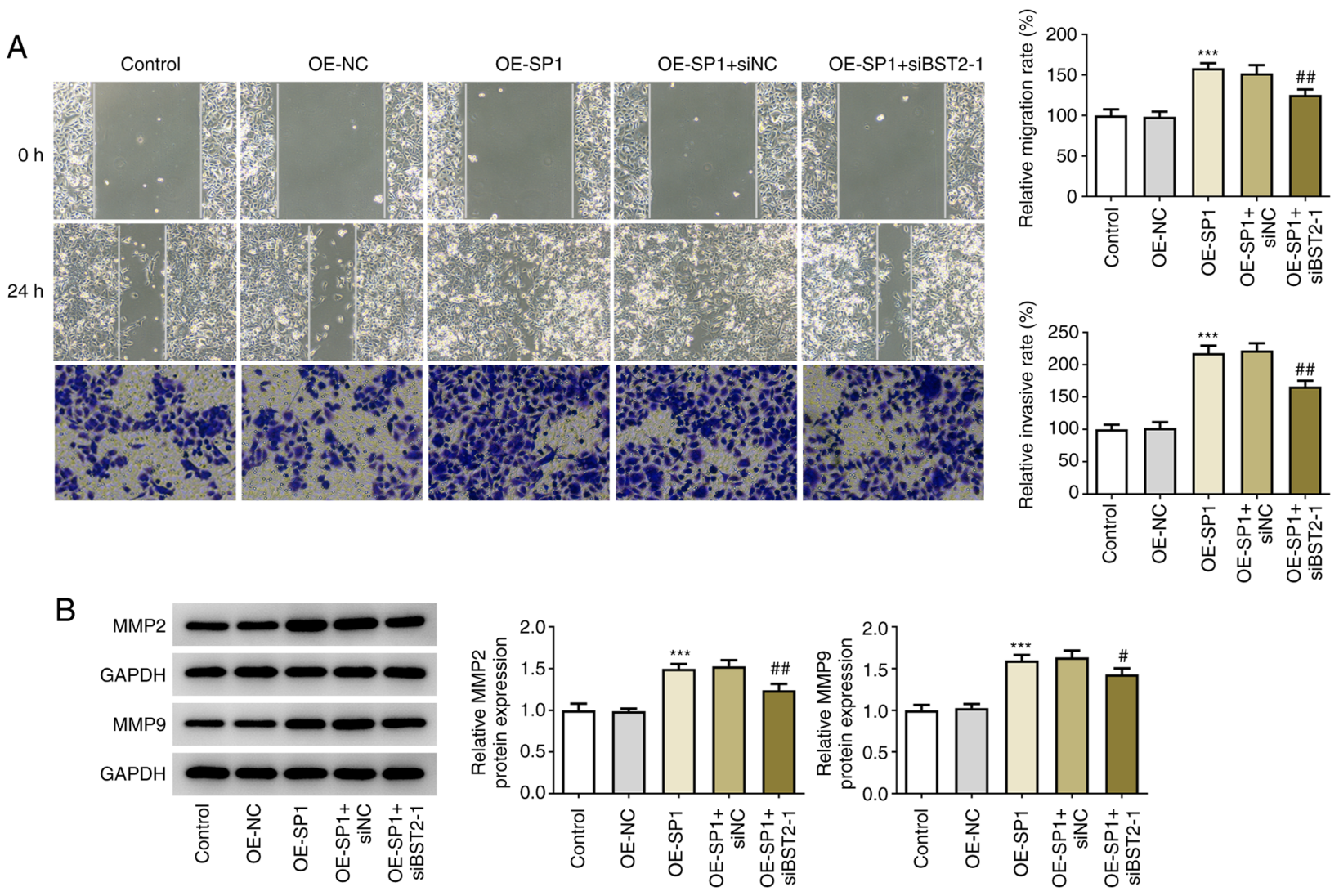

Figure 5. BST2 knockdown reverses SP1 overexpression-induced cell migration. (A) Analysis of PANC1 cell migration and invasion by wound healing (top and middle rows) and Transwell (bottom row) assays, respectively, after transfection with OE-NC/OE-SP1 or co-transfection with OE-SP1 and siNC/siBST2-1 (magnification, x100). (B) Expression of migration markers assayed by western blotting. ${ }^{* * *} \mathrm{P}<0.001 \mathrm{vs}$. OE-NC; ${ }^{\#} \mathrm{P}<0.05$, ${ }^{\# \#} \mathrm{P}<0.01 \mathrm{vs}$. OE-SP1 + siNC. SP1, specificity protein 1; BST2, bone marrow stromal cell antigen 2; OE, overexpression; NC, negative control; si, small interfering RNA.

In the present study, the results analyzed in the GEPIA database also showed a direct association between BST2 expression and pancreatic cancer. Upregulated BST2 expression was observed in all four pancreatic cancer cell lines compared with the non-cancerous pancreatic duct epithelial cells. Moreover, multiple pathways and interactive activities have been explored in previous studies to determine the mechanisms of action of BST2 in cancer progression. Xu et al (26) investigated the role of BST2 in hepatocellular carcinoma (HCC) and found that high BST2 expression in HCC tissues was positively associated with tumor growth, possibly via activation of the NF- $\kappa \mathrm{B}$ pathway. Liu et al (27) demonstrated that BST2 downregulation by microRNA (miR)-760 was favorable for the repression of gastric cancer cell survival and migration. An earlier study by Liu et al (28) also elucidated the association between BST2-induced NF- $\mathrm{KB}$ activation and increased proliferation and migration of gastric cancer cells. The present study demonstrated that knockdown of BST2 in the PANC1 pancreatic cancer cell line effectively mitigated the proliferative and migratory cell behaviors.

The JASPAR database of transcription factor binding prediction was consulted to describe the action of BST2 on PANC1 cell proliferation and migration from a mechanistic perspective. The transcription factor SP1, a proven oncogene in various types of tumors, was predicted to have a matching sequence on the BST2 promoter. The results of the ChIP and dual-luciferase reporter assays in the present study verified the binding relationship between SP1 and the BST2 promoter. Zhang et al (29) reported that the oncogenic effects of SP1 in HCC in vitro were mediated through transcriptional upregulation of RAS guanyl-releasing protein 1 . Targeted inhibition of SP1 by miR-502-5p in gastric cancer cells decreased the levels of cell proliferation, invasion and migration (30). Moreover, in a previous study, SP1 was found to be targeted by miR-529, thereby suppressing the malignant behaviors and epithelial-to-mesenchymal transition of pancreatic cancer cells (31). IncRNA-LINC00514 upregulation mediated via SP1 acts as an oncogene in metastatic osteosarcoma by regulating miR-708 expression (32). As a transcription factor, SP1 activates IncRNA SNGH7 to promote ovarian tumorigenesis (33). The present study demonstrated SP1 overexpression-induced PANC1 cell proliferation and migration, while BST2 knockdown weakened this effect of SP1 overexpression.

The results of the present study supplemented the current understanding of the role of BST2 in cancer progression with corroborating evidence of increased proliferation and migration of pancreatic cancer cells via SP1-activated BST2. The findings of the present study may provide a useful guideline for the identification of the prognostic and therapeutic value of SP1 and BST2 in future relevant research and clinical trials. However, a limitation of this study was the insufficient discussion on the downstream regulatory mechanisms of BST2. Therefore, further research is required in the future. 


\section{Acknowledgements}

Not applicable.

\section{Funding}

The present study was financially supported by the National Nature Science Foundation of China (grant no. 81071985).

\section{Availability of data and materials}

The datasets used and/or analyzed during the current study are available from the corresponding author on reasonable request.

\section{Authors' contributions}

CL, YH and JC conceived and designed the study, and acquired and interpreted the data. CL was a major contributor in writing the manuscript. All authors confirm the authenticity of all the raw data. All authors have read and approved the final manuscript.

\section{Ethics approval and consent to participate}

Not applicable.

\section{Patient consent for publication}

Not applicable.

\section{Competing interests}

The authors declare that they have no competing interests.

\section{References}

1. Vincent A, Herman J, Schulick R, Hruban RH and Goggins M: Pancreatic cancer. Lancet 378: 607-620, 2011.

2. Bray F, Ferlay J, Soerjomataram I, Siegel RL, Torre LA and Jemal A: Global cancer statistics 2018: GLOBOCAN estimates of incidence and mortality worldwide for 36 cancers in 185 countries. CA Cancer J Clin 68: 394-424, 2018.

3. Ilic M and Ilic I: Epidemiology of pancreatic cancer. World J Gastroenterol 22: 9694-9705, 2016.

4. Siegel RL, Miller KD and Jemal A: Cancer statistics, 2019. CA Cancer J Clin 69: 7-34, 2019.

5. Mizrahi JD, Surana R, Valle JW and Shroff RT: Pancreatic cancer. Lancet 395: 2008-2020, 2020.

6. Qian L, Yu S, Chen Z, Meng Z, Huang S and Wang P: Functions and clinical implications of exosomes in pancreatic cancer. Biochim Biophys Acta Rev Cancer 1871: 75-84, 2019.

7. Zhang L, Sanagapalli S and Stoita A: Challenges in diagnosis of pancreatic cancer. World J Gastroenterol 24: 2047-2060, 2018.

8. Yang J, Ren B, Yang G, Wang H, Chen G, You L, Zhang T and Zhao Y: The enhancement of glycolysis regulates pancreatic cancer metastasis. Cell Mol Life Sci 77: 305-321, 2020.

9. Rouanet M, Lebrin M, Gross F, Bournet B, Cordelier P and Buscail L: Gene Therapy for Pancreatic Cancer: Specificity, Issues and Hopes. Int J Mol Sci 18: 1231, 2017.

10. Kurtanich T, Roos N, Wang G, Yang J, Wang A and Chung EJ: Pancreatic Cancer Gene Therapy Delivered by Nanoparticles. SLAS Technol 24: 151-160, 2019.

11. Wu J, Li Z, Zeng K, Wu K, Xu D, Zhou J and Xu L: Key genes associated with pancreatic cancer and their association with outcomes: A bioinformatics analysis. Mol Med Rep 20: 1343-1352, 2019.
12. Arnaud F, Black SG, Murphy L, Griffiths DJ, Neil SJ, Spencer TE and Palmarini M: Interplay between ovine bone marrow stromal cell antigen 2/tetherin and endogenous retroviruses. J Virol 84: 4415-4425, 2010.

13. Gong S, Osei ES, Kaplan D, Chen YH and Meyerson H: CD317 is over-expressed in B-cell chronic lymphocytic leukemia, but not B-cell acute lymphoblastic leukemia. Int J Clin Exp Pathol 8: 1613-1621, 2015.

14. Wang W,Nishioka Y,Ozaki S,Jalili A,AbeS, Kakiuchi S, KishukuM, Minakuchi K, Matsumoto T and Sone S: HM1.24 (CD317) is a novel target against lung cancer for immunotherapy using anti-HM1.24 antibody. Cancer Immunol Immunother 58: 967-976, 2009.

15. Malsy M, Graf B and Almstedt K: The active role of the transcription factor Sp1 in NFATc2-mediated gene regulation in pancreatic cancer. BMC Biochem 20: 2, 2019.

16. Safe S, Nair V and Karki K: Metformin-induced anticancer activities: Recent insights. Biol Chem 399: 321-335, 2018.

17. Nair V, Pathi S, Jutooru I, Sreevalsan S, Basha R, Abdelrahim M, Samudio I and Safe S: Metformin inhibits pancreatic cancer cell and tumor growth and downregulates Sp transcription factors. Carcinogenesis 34: 2870-2879, 2013.

18. Livak KJ and Schmittgen TD: Analysis of relative gene expression data using real-time quantitative PCR and the 2(-Delta Delta C(T)) Method. Methods 25: 402-408, 2001.

19. Chen W, Zheng R, Baade PD, Zhang S, Zeng H, Bray F, Jemal A, Yu XQ and He J: Cancer statistics in China, 2015. CA Cancer J Clin 66: 115-132, 2016.

20. Hogendorf P, Durczyński A and Strzelczyk J: Metastatic Pancreatic Cancer. J Invest Surg 31: 151-152, 2018.

21. Ferrone CR, Brennan MF, Gonen M, Coit DG, Fong Y, Chung S, Tang L, Klimstra D and Allen PJ: Pancreatic adenocarcinoma: The actual 5-year survivors. J Gastrointest Surg 12: 701-706, 2008.

22. Hackert T, Klaiber U, Pausch T, Mihaljevic AL and Büchler MW: Fifty Years of Surgery for Pancreatic Cancer. Pancreas 49: 1005-1013, 2020.

23. Das S and Batra SK: Pancreatic cancer metastasis: Are we being pre-EMTed? Curr Pharm Des 21: 1249-1255, 2015.

24. Ishikawa J, Kaisho T, Tomizawa H, Lee BO, Kobune Y, Inazawa J, Oritani K, Itoh M, Ochi T, Ishihara K, et al: Molecular cloning and chromosomal mapping of a bone marrow stromal cell surface gene, BST2, that may be involved in pre-B-cell growth. Genomics 26 : 527-534, 1995.

25. Fujiwara K, Ohuchida K, Sada M, Horioka K, Ulrich CD III, Shindo K, Ohtsuka T, Takahata S, Mizumoto K, Oda Y, et al: CD166/ALCAM expression is characteristic of tumorigenicity and invasive and migratory activities of pancreatic cancer cells. PLoS One 9: e107247, 2014.

26. Xu X, Wang Y, Xue F, Guan E, Tian F, Xu J and Zhang H: BST2 Promotes Tumor Growth via Multiple Pathways in Hepatocellular Carcinoma. Cancer Invest 38: 329-337, 2020.

27. Liu W, Li Y, Feng S, Guan Y and Cao Y: MicroRNA-760 inhibits cell viability and migration through down-regulating BST2 in gastric cancer. J Biochem 168: 159-170, 2020.

28. Liu W, Cao Y, Guan Y and Zheng C: BST2 promotes cell proliferation, migration and induces NF- $\mathrm{KB}$ activation in gastric cancer. Biotechnol Lett 40: 1015-1027, 2018.

29. Zhang X, Zhuang H, Han F, Shao X, Liu Y, Ma X, Wang Z, Qiang Z and Li Y: Spl-regulated transcription of RasGRP1 promotes hepatocellular carcinoma (HCC) proliferation. Liver Int 38: 2006-2017, 2018.

30. Peng X, Wu M, Liu W, Guo C, Zhan L and Zhan X: miR-502-5p inhibits the proliferation, migration and invasion of gastric cancer cells by targeting SP1. Oncol Lett 20: 2757-2762, 2020.

31. Xue L, Shen Y, Zhai Z and Zheng S: miR 539 suppresses the proliferation, migration, invasion and epithelial mesenchymal transition of pancreatic cancer cells through targeting SP1. Int J Mol Med 45: 1771-1782, 2020.

32. Mi LD, Sun CX, He SW and Du GY: SP1-Induced Upregulation of lncRNA LINC00514 Promotes Tumor Proliferation and Metastasis in Osteosarcoma by Regulating miR-708. Cancer Manag Res 12: 3311-3322, 2020.

33. Bai Z, Wu Y, Bai S, Yan Y, Kang H, Ma W, Zhang J, Gao Y, Hui B, Ma H, et al: Long non-coding RNA SNGH7 Is activated by SP1 and exerts oncogenic properties by interacting with EZH2 in ovarian cancer. J Cell Mol Med 24: 7479-7489, 2020.

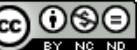

This work is licensed under a Creative Commons Attribution-NonCommercial-NoDerivatives 4.0 International (CC BY-NC-ND 4.0) License. 